\section{G37(P) LABORATORY CORRELATES OF SEVERITY IN ACUTE BRONCHIOLITIS OF INFANCY}

1,2M Ghazaly, ${ }^{3} \mathrm{R}$ Thwaites, ${ }^{1} \mathrm{~K}$ Feather, ${ }^{4} \mathrm{~K}$ Ito, ${ }^{4} \mathrm{G}$ RapePort, $3,5 \mathrm{p}$ Openshaw, ${ }^{3,5} \mathrm{~T}$ Hansel, 1,5S Nadel. 'Paediatric Intensive Care Unit, St Mary's Hospital, Imperial College Healthcare Trust, London, UK; ${ }^{2}$ Pediatric Department, Faculty of Medicine, Assiut University, Assiut, Egypt; ${ }^{3}$ National Heart and Lung Institute, Faculty of Medicine, Imperial College London, London, UK; ${ }^{4}$ Pulmocide Ltd, London, UK; ${ }^{5}$ Faculty of Medicine, Imperial College London, London, UK

\subsection{6/archdischild-2018-rcpch.35}

Introduction Respiratory viral infections cause significant morbidity and mortality in infants; approximately half of which are associated with respiratory syncytial virus (RSV) infection. Severe bronchiolitis has been associated with an aberrant host response to viral replication. We investigated the patient characteristics, immune response and viral load in bronchiolitis.

Methods We recruited patients $<2$ years of age presenting to St Mary's Hospital between October 2016-March 2017 with a clinical diagnosis of bronchiolitis. We recruited 55 hospitalised babies; grouped into moderate $(n=34)$ and severe $(n=21)$ based on their need for invasive respiratory support. Respiratory samples were collected up to twice daily using nasosorption and nasopharyngeal aspiration (NPA) along with severity scores (PEWs, M-WCAS, and RESVINET). Viral load was determined by qPCR (Primer Design, UK), immune mediators were quantified by MSD (MSD, Rockville, USA).

Results Younger age, lower weight and more co-morbidities were observed among severe cases; $50 \%$ had apnoea on presentation. Taking disease duration into account, viral load was observed to be significantly lower in severe RSV cases, compared to moderate cases, although decline in viral load was quicker (table 1).

Levels of IFN- $\gamma$ and CXCL10/IP-10 were significantly higher in moderate versus severe cases. Levels of many inflammatory mediators (including type-II cytokines) were not significantly different between groups whilst mucin levels were elevated in severe cases.

Conclusion A strong innate anti-viral immune response early in disease may protect against severe RSV disease, despite higher viral load. Detailed study of airway mucosal responses to infection may clarify determinants of severity in bronchiolitis.

\begin{tabular}{llll}
$\begin{array}{l}\text { Abstract G37(P) Table 1 } \\
\text { infants with viral bronchiolitis }\end{array}$ & Clinical characteristics and virology in \\
\hline & $\begin{array}{l}\text { Moderate cases } \\
(\mathbf{n}=34)\end{array}$ & $\begin{array}{l}\text { Severe cases } \\
(\mathbf{n}=21)\end{array}$ & P-value \\
\hline Age (days)median (range) & $180(30-630)$ & $56(15-630)$ & $0.028^{*}$ \\
Sex (male:female) & $21: 13$ & $13: 8$ & $>0.999$ \\
Wt (kg) median (range) & $7.3(4.38-12.8)$ & $3.9(2.2-13.7)$ & $0.0057^{*}$ \\
Prematurity n (\%) & $11(32)$ & $7(33)$ & $>0.999$ \\
Other co morbidity n (\%) & $11(32)$ & $15(71)$ & $0.006^{*}$ \\
Length of hospital stay median (range) & $2(0.5-7)$ & $14(3-38)$ & $<0.0001^{*}$ \\
Time between symptoms and admission & $3(0-15)$ & $3(0-15)$ & 0.33 \\
median (range) & & $12(57)$ & 0.788 \\
RSV positive $n$ (\%) & $18(53)$ & & \\
\hline
\end{tabular}

\section{G38(P) HOW MUCH IS THAT DOGGY IN THE PICU? THE ROLE OF A REGISTERED THERAPY DOG IN PAEDIATRIC INTENSIVE CARE}

${ }^{1} \mathrm{~S}$ Emsden, ${ }^{1,2} \mathrm{C}$ Kanaris. 'North West and North Wales Paediatric Transport Service, Warrington, UK; ${ }^{2}$ The Centre for Social Ethics and Policy, School of Law, The University of Manchester, Manchester, UK

\subsection{6/archdischild-2018-rcpch.36}

The objective of the paper is to share our experiences using a Therapy Dog in our service and to demonstrate the benefits it can bring to patients and staff.

Animals can play a valuable part in alleviating stress in many situations. Paediatric Intensive Care Medicine poses a unique set of challenges, not only in terms of clinical management but also in terms of the amount of emotional support that patients, their families and staff members require. The North West and North Wales Paediatric Transport Service (NWTS) is a specialised team that deals with the stabilisation and transfer of critically ill children. Communicating with families during transit and after arrival to PICU can be challenging, clinical information needs to be relayed during a very stressful period. To address this problem, we have produced a photo book for families to read to their children. It tells the story of a curious pug, Alfie, who is invited to join the NWTS team to find out what happens when a child is ill and needs to go to intensive care.

Alfie is a therapy dog registered with Pets As Therapy UK, he has been assisting the critical care team in communicating with children and their families. He also has sessions whereby staff members who need help and support coping with stressful situations can spend time with him. Alfie also frequently visits the PICU and wards at the Royal Manchester Children's Hospital and offers support to numerous children undergoing treatment.

In order to assess how the information booklet was perceived and the impact that Alfie's sessions have had on individuals we handed out feedback questionnaires to families and staff. The feedback was universally positive, leading to the conclusion that a registered therapy animal can be beneficial to both staff and patients in a PICU setting.

\section{G39(P) A TEENAGER WITH A PANIC ATTACK AND HOW IT UNFOLDED}

LHW Saych, P Desai. Paediatrics, Ipswish Hospital, Ipswich, UK

10.1136/archdischild-2018-rcpch.37

A 14 year old boy presented to a Paediatric Assessment Unit with shortness of breath and chest pain. He had never been seen in primary or secondary care before. His first medical attendance was to $\mathrm{A}$ and $\mathrm{E}$ with these symptoms 8 days earlier, when he was started on amoxicillin for a suspected chest infection. He was pale, tachypnoeic and hypertensive. The initial impression was that he might be having a panic attack. 
He described 9 days of breathlessness, chest tightness, vomiting and abdominal pain. His chest was clear and heart sounds normal with a soft murmur. He appeared very anxious. There were two positive findings of significant concern; a 3 centimetre palpable liver and persistent hypertension of more than 160/120. A chest radiograph showed a large heart and fluid in the horizontal fissure. More focused history taking revealed the shortness of breath was worse on exertion and on lying down, and he had to prop himself up at night to sleep.

The history and examination was consistent with heart failure. An ECG showed right ventricular hypertrophy and widespread T-wave inversion. An echocardiogram showed a structurally normal heart, mild mitral and aortic regurgitation, severely impaired left ventricular function (fractional shortening 13\%) and bilateral pleural effusions. A provisional diagnosis of viral myocarditis was made. However, in addition to persistent hypertension, his urea was 30 and creatinine 523.

He was admitted to a Paediatric Intensive Care Unit where acute management included oxygen, fluid restriction, furosemide and a milrinone infusion. In view of the deranged renal profile he was discussed with the renal team. Renal ultrasound revealed small, scarred kidneys and calyceal dilatation. The diagnosis was end stage renal failure due to undiagnosed grade 4 vesico-ureteric reflux, hypertension secondary to chronic kidney disease, and dilated cardiomyopathy secondary to hypertension.

It is rare for a teenager to present in heart failure, having never been unwell before. Originally we thought this was a primary cardiac problem, but the learning point here is that despite antenatal screening and good health care, congenital renal disease can remain asymptomatic, and that end stage renal failure can present with heart failure.

\section{G40(P) IS THERE ROLE FOR ECULIZUMAB IN THE MANAGEMENT OF C3 GLOMERULOPATHY?}

${ }^{1} \mathrm{D}$ Stewart, ${ }^{1} \mathrm{D}$ Hughes, ${ }^{2} \mathrm{~L}$ Riach, ${ }^{2} \mathrm{C}$ Oxley, ${ }^{1} \mathrm{D}$ Athavale. ${ }^{1}$ Renal Unit, Royal Hospital for Children, Glasgow, UK; 'Paediatric Department, Royal Aberdeen Children's Hospital, Aberdeen, UK

\subsection{6/archdischild-2018-rcpch.38}

Introduction The new classification, and increasing recognition, of dense deposit disease (DDD) as a C3 glomerulopathy and an alternative complement pathway disorder suggests that eculizumab, an anti-C5 monoclonal antibody, may be beneficial in treatment. However, the use of eculizumab as an off-label and expensive treatment in paediatrics is limited with variable clinical response in literature.

Method We present the outcomes of 2 paediatric patients in Scotland who have received eculizumab for the management of DDD.

Results Case 1: A 10-year-old male presented with steroidresistant proteinuria and biopsy confirmed DDD. He was managed symptomatically with an angiotensin converting inhibitor and diuretics. Four years later, due to worsening symptoms (oedema, lethargy, poor growth), renal function deterioration, and elevated terminal complement complex (TCC) levels, eculizumab was commenced. Repeat biopsy demonstrated chronic irreversible damage but 25\% glomerular architecture preservation. Symptomatic improvement was seen along with a reduction in proteinuria and renal function stabilisation. After 24 months, eculizumab was discontinued with progressive renal function deterioration seen in the final months of therapy. Six months later, due to worsening of symptoms and increasing proteinuria, eculizumab was recommenced with symptomatic improvement noted.

Case 2: A 5-year-old girl presented with steroid-resistant haematuria and proteinuria and biopsy confirmed DDD. Sixteen months following diagnosis, she rapidly progressed towards end-stage renal disease. Haemodialysis was commenced following no benefit from immunosuppression (mycophenolate mofetil or plasmapheresis). TCC level was elevated and eculizumab treatment was commenced 18 months after diagnosis. Renal biopsy prior to treatment showed marked pathological changes in all visible glomeruli. However, due to lack of clinical response, eculizumab was discontinued after 2 months. The patient remaining dialysis dependent.

Conclusions Our experience with eculizumab has demonstrated variable results. Only one patient exhibited a positive clinical response. Eculizumab was administered to both patients at varying points in their clinical journey with differing progressive biopsy features. Our experience adds to the limited evidence in literature, suggesting that a role for eculizumab in DDD may be guided by clinical and pathological features. However, further trials of its use in paediatric DDD are clearly needed.

\section{British Inherited Metabolic Diseases Group and British Society of Paediatric Gastroenterology, Hepatology and Nutrition}

\section{G41 A JOINT COLLABORATIVE REGIONAL NETWORK APPROACH TO OVERCOME DIFFICULTIES IN DIAGNOSIS OF PAEDIATRIC COELIAC DISEASE}

${ }^{1}$ A Hakizimana, ${ }^{2} \mathrm{~N}$ Heather, ${ }^{3} \mathrm{~J}$ Pridgeon, ${ }^{4} \mathrm{~N}$ Ward, ${ }^{2} \mathrm{NA}$ Afzal. ${ }^{1}$ Faculty of Medicine, University of Southampton, Southampton, UK; ${ }^{2}$ Department of Paediatrics, Southampton Children's Hospital, Southampton, UK; ${ }^{3}$ Department of Paediatrics, Queen Alexandra Hospital, Portsmouth, UK; ${ }^{4}$ Department of Paediatrics, Hampshire Hospitals NHS Foundation Trust, Basingstoke, UK

\subsection{6/archdischild-2018-rcpch.39}

Aims Diagnosis of paediatric coeliac disease (pCD) continues to be a challenge despite the new NICE, BSPGHAN and ESPGHAN guidance. To understand a 'true abnormal serologic result' and the need for a duodenal biopsy, we aimed for a quality improving exercise in a regional network setting.

Methods A two staged quality approach was adopted:

- An online regional survey was conducted to investigate the currently used serologic screening tests for diagnosis of pCD in the region. Regional paediatricians and pathology laboratories were contacted by phone and email followed by an online survey conducted via the Network Website (AH, NA).

- A summary presentation of the findings, were presented at the annual regional network meeting followed by a rigorous discussion with active participation of immunologists, paediatric consultants and dieticians from the region.

Results 9/13 hospitals in the regional network participated in the survey.

For coeliac screening IgA Anti-tTG is used by all centres (IgA EMA in $7 / 9$ centres (77.8\%). 5/9 (55.6\%) centres routinely check IgA levels in all cases. $5 / 9$ centres $(55.6 \%)$ combined 